Documentation et bibliothèques

DOCUMENTATION BIBLIOTHEQUES

\title{
Les 25 ans de SDM : une histoire à suivre
}

The 25 Years of SDM: A Story to Watch

Los 25 años de SDM: una historia que seguir

\section{Paul Francoeur}

Volume 36, numéro 1, janvier-mars 1990

URI : https://id.erudit.org/iderudit/1028197ar

DOI : https://doi.org/10.7202/1028197ar

Aller au sommaire du numéro

\section{Éditeur(s)}

Association pour l'avancement des sciences et des techniques de la documentation (ASTED)

ISSN

0315-2340 (imprimé)

2291-8949 (numérique)

Découvrir la revue

Citer cet article

Francoeur, P. (1990). Les 25 ans de SDM : une histoire à suivre. Documentation et bibliothèques, 36(1), 5-9. https://doi.org/10.7202/1028197ar
Résumé de l'article

L'auteur tente de dégager certaines caractéristiques des Services documentaires multimedia (SDM), organisme unique dans le monde francophone. Des débuts quelque peu incertains jusqu'à la reconnaissance finale de la qualité de sa gestion administrative et professionnelle par tous les milieux bibliothéconomiques tant québécois, canadiens qu'étrangers, SDM a diversifié ses services et ses marchés pour assurer son avenir : choix et traitement des livres, des documents audiovisuels, des périodiques, des jeux et jouets éducatifs, des logiciels.
Tous droits réservés $@$ Association pour l'avancement des sciences et des techniques de la documentation (ASTED), 1990
Ce document est protégé par la loi sur le droit d'auteur. L’utilisation des services d'Érudit (y compris la reproduction) est assujettie à sa politique d'utilisation que vous pouvez consulter en ligne.

https://apropos.erudit.org/fr/usagers/politique-dutilisation/ 


\title{
Les 25 ans de SDM : une histoire à suivre
}

\author{
Paul Francoeur* \\ Ministère de l'Éducation du Québec
}

L'auteur tente de dégager certaines caractéristiques des Services documentaires multimedia (SDM), organisme unique dans le monde francophone. Des débuts quelque peu incertains jusqu'à la reconnaissance finale de la qualité de sa gestion administrative et professionnelle par tous les milieux bibliothéconomiques tant québécois, canadiens qu'étrangers, SDM a diversifié ses services et ses marchés pour assurer son avenir: choix et traitement des livres, des documents audiovisuels, des périodiques, des jeux et jouets éducatifs, des logiciels.

\section{The 25 Years of SDM: A Story to Watch}

The author identifies the characteristics of the Services documentaires multimedia (SDM), a unique organisation in the francophone world. From its shaky beginning to the recognition of its administrative and professional management by libraries in Québec, in Canada and elsewhere, SDM has developed its services and its markets in order to insure its future: selection and cataloguing of books, audiovisual material, periodicals, games and educational toys, and software.

\section{Los 25 años de SDM: una historia que seguir}

El autor trata de destacar ciertas características de los Servicios Documentales Multimedia (SDM), organismo único en el mundo de habla francesa. Desde sus comienzos inciertos hasta el reconocimiento final de la calidad de su gestión administrativa y profesional en todos los medios biblioteconómicos tanto quebequenses como canadienses o extranjeros, SDM ha diversificado sus servicios y sus mercados para asegurar su porvenir: selección y tratamiento de libros, de documentos audiovisuales, de periódicos, de juegos y juguetes educativos y también de programas y sistemas de programación.
Au nord de Montréal, dans le quartier du Saultau-Récollet, discrètement blottis à l'étage d'un immeuble en brique bâti près de l'ancien jardin du noviciat des Jésuites, logent les Services documentaires multimedia (SDM), entreprise héritière de la Centrale des bibliothèques (CB).

Dans le rayonnement des festivités qui ont entouré le $25^{\mathrm{e}}$ anniversaire du ministère de I'Éducation, SDM, qui est l'un de ses vigoureux surgeons, pavoise pour son propre compte avec une légitime fierté et met en évidence plus d'un quart de siècle de contribution marquante au développement de l'information documentaire au Québec.

Nous tenterons ici, dans un bref survol historique, de dégager à grands traits certaines caractéristiques de cet organisme dont on ne trouve pas d'exemple équivalent dans le monde francophone. On permettra à celui qui fut trop longtemps associé à sa fondation et à son développement, soit du $1^{\text {er }}$ avril 1963 au $1^{\text {er }}$ avril 1986, de se livrer à cet exercice en faisant aussi appel à la mémoire du coeur et en mettant à profit le relief engendré par le temps et l'éloignement.

\section{Une longue difficulté d'être}

En 1963, la conception d'une Centrale s'élaborait sous de précaires auspices. Les promoteurs du projet n'avaient ni la conscience ni l'ambition de jeter les bases d'une institution durable. IIs entendaient plutôt susciter un service d'appoint qui, à moyen terme, favoriserait l'essor des bibliothèques de collège.

La Fédération des collèges classiques qui, en septembre 1964, donnait naissance à une cen-

\footnotetext{
* L'auteur est responsable des bibliothèques scolaires à la Direction régionale de la Montérégie au Ministère de l'Éducation du Québec. 
trale de catalogage n'entretenait pas d'illusions sur les perspectives de sa propre survie. Le rejeton aurait bien vite à voler de ses propre ailes, compte tenu des recommandations du Rapport Parent dont l'application s'amorçait.

Dans ce contexte d'incertitude, la nouvelle de cette naissance fut assez mal reçue par les éminences grises qui planifiaient à l'époque les principales institutions bibliothéconomiques dont le Québec allait se doter à la faveur de la Révolution tranquille. La jeune Centrale, inattendue et dangereusement vigoureuse, menaçait la cohérence du programme d'ensemble. Edmond Desrochers, l'un des plus illustres représentants de ce groupe, engagea loyalement un combat redoutable contre cette excroissance indue, hostilité qui prit fin en 1967 avec l'entrée de la Centrale des bibliothèques dans le giron du nouveau ministère de l'Éducation.

Au même moment parvenait de France une nouvelle sombre: I'Administration des bibliothèques sabrait dans les services bibliographiques officiels dirigés par Marie-Noëlle Malclès. Typique exemple du désintérêt de certains grands commis de l'État pour ce genre d'activité d'intérêt national.

Pendant presque vingt ans, la Centrale des bibliothèques vécut pratiquement sans existence propre, sans dénomination précise, avec un statut précaire, sous tutelle administrative: d'abord comme un service de la Fédération des collèges classiques, ensuite comme division du Service des bibliothèques d'enseignement, puis du Service général des moyens d'enseignement. L'appellation "Centrale des bibliothèques" ne constituait qu'une étiquette ne recouvrant aucune réalité juridique. Celle-ci lui fut accordée en 1982, au moment de l'incorporation.

Au début des années 70 , certains décrétèrent sa mission désuète avec l'avènement de l'audiovisuel et la fin présumée de l'imprimé. Un scénario semblable se répéta au début des années 80 avec l'arrivée de l'informatique.

Mais la menace la plus insidieuse vint, dans les années 70 , des contraintes financières de l'État qui allaient peu à peu anémier ou asphyxier certaines catégories de bibliothèques qui représentaient sa clientèle principale.

«Et pourtant, elle vit!» serait-on tenté de pasticher à la suite de Galilée.

\section{Une place au soleil}

Au milieu de ces vicissitudes, la Centrale des bibliothèques connut une croissance ferme et constante, si bien qu'aujourd'hui elle lègue à SDM une situation enviable de permanence, de stabilité, d'avenir.

Sa raison d'être originelle s'est révélée beaucoup plus profonde et large que prévu. Après avoir piétiné quelque temps les plates-bandes d'organismes apparentés - Bibliothèque nationale du Québec, Bureau des bibliothèques scolaires, Service des bibliothèques publiques, École de bibliothéconomie, associations professionnelles, bibliothèques universitaires, entreprises spécialisées - elle se concentra sur un créneau qui devint son apanage et que nul ne lui conteste maintenant.

Avouons-le, cette évolution emprunta parfois des allures de conquête. II n'y avait place au Québec que pour un seul service de ce genre. Les impératifs de viabilité commandaient la formation d'une sorte de monopole. Fusions, annexions, concentrations, ententes, intégrations, unions, absorptions, assimilations jalonnent son histoire. Ce processus se déroula assez pacifiquement, quoique parfois les ukases de gestionnaires en mal de rationalisation donnèrent le coup de pouce.

Tout au long de cette évolution féconde, la Centrale des bibliothèques a rarement défrayé la manchette de l'actualité. Peu encline aux petites intrigues, aux jeux de coulisses, au lobbying, elle a par ailleurs modestement investi dans les relations publiques. Elle fréquenta rarement les manifestations de prestige et se fit discrète sur les tribunes nationales et internationales. À bon vin point d'enseigne.

Le meilleur de son énergie, elle le réserva au travail quotidien, acharné, persévérant, misant sur la qualité et la pertinence de ses productions, gardant le cap sur le long terme, consciente d'avoir à fournir une oeuvre de longue haleine.

Ce pari a donné des dividendes: SDM a pignon sur rue; sa raison d'être est socialement, culturellement et économiquement incontestable; un équilibre harmonieux s'est créé avec les organismes complémentaires; la valeur de ses travaux est largement reconnue; l'entreprise se prépare activement à relever les défis du $\mathrm{XXI}^{\mathrm{e}}$ siècle.

\section{Un parti pris d'excellence}

Au fil des ans, la Centrale des bibliothèques s'attirera à tort ou à raison une variété de reproches: coût de production trop élevé, retard chronique dans le traitement des nouveautés de l'édition, indifférence à l'endroit des besoins de la pratique pédagogique, arrogance d'une organisation monopolistique, tendance à faire bande 
à part, propension au conservatisme, etc. Mais presque jamais on n'a mis en doute la valeur du travail fourni et la qualité des sous-produits qui en découlent.

Une fois dépassée l'étape dite artisanale des commencements, la Centrale s'est hissée et s'est maintenue dans son domaine au niveau des plus exigeantes normes internationales. L'informatisation affermit cette caractéristique en assurant la mise à jour et la cohérence de centaines de milliers de notices qui s'étaient stratifiées d'année en année.

Cette qualité a prévalu dans les différents secteurs où la Centrale des bibliothèques a exercé son action; elle lui sert encore d'image de marque. À long terme, ce souci de perfection lui a valu la confiance des administrateurs et des usagers.

Le mérite en revient surtout aux membres du personnel de SDM dont la valeur professionnelle et technique dépasse largement la moyenne. Historiquement, la Centrale s'est trouvée au noeud d'un réseau exceptionnel de relations. Longtemps, elle disposa des arguments et des moyens de recruter des candidats de grand calibre, écrémant ainsi les milieux de la documentation. Heureux de s'intégrer à une équipe dynamique, les nouveaux venus pouvaient acquérir un supplément de formation sur place, étant donné la nature particulière des travaux exécutés au sein de l'entreprise.

À peu d'exceptions près, les recruteurs eurent la main heureuse. La majorité des personnes embauchées se révélèrent bien adaptées à cette tâche et s'incorporèrent avec facilité. Le travail quotidien à SDM requiert une aptitude à l'application constante, la faculté de surmonter I'ennui d'une routine quotidienne, dans un climat de calme et de silence relatifs. Des visiteurs occasionnels se sont étonnés du caractère insolite de cette ruche doucement bourdonnante qu'on a parfois qualifiée de "monastère de la rue Fleury".

Ce personnel de choix a dans l'ensemble fait preuve d'une stabilité remarquable que seuls la maladie, la retraite ou le décès ne venaient troubler. On a de plus enregistré un nombre limité de rejets.

Ce groupe de personnes bien identifiées à l'entreprise a toujours fait preuve de loyauté et d'un grand sens de responsabilité à l'endroit de leur communauté de travail. À l'heure de la syndicalisation, quand le temps fut venu de passer d'un régime familial et paternaliste à un système de relations de travail mieux structuré, la collectivité fit preuve d'une bonne maturité.
Saluons au passage quelques membres de l'encadrement, anciens et actuels, qui ont particulièrement contribué à créer et à perpétuer cette tradition d'excellence de SDM:

- Raymonde Couture: maître en cartographie, sa capacité de travail hors du commun, alliée à une compétence et à une expérience uniques, a conféré à une myriade de notices bibliographiques une perfection inégalée ;

- Aloïs Gutzwiller: homme de grande culture, humaniste raffiné, il donna à l'activité bibliographique un essor incomparable ;

- Jacques Aird: de formation scientifique et réputé pour la rigueur de sa méthode de travail, il améliora de façon notable le fonctionnement général de l'organisme et ouvrit la voie de l'informatisation;

- Alain Boucher: esprit de grande envergure, il lui revient d'avoir façonné le visage moderne de la Centrale, en lui faisant prendre avec succès le délicat virage technologique;

- Jacques Demers: dans un milieu dominé par l'imprimé, il forgea avec doigté et persévérance un petit empire de la documentation audiovisuelle;

- Diane Dallaire: jeune recrue brillante qui gravit rapidement les échelons de l'administration; elle apporta une contribution précieuse à la transparence de la gestion et à l'établissement de rapports humains confiants avec le personnel.

\section{Sous le signe de la Vierge}

Née à l'équinoxe de l'automne 1964, sous le beau signe de la Vierge, la Centrale a sûrement profité d'une heureuse conjonction astrale puisqu'elle a surmonté victorieusement la plupart des embûches dressées sur sa route et qu'elle a toujours trouvé à point nommé les appuis tutélaires requis par la situation.

II est significatif de récapituler la liste de ses principaux protecteurs.

Au commencement, on trouve réunie autour de son berceau et de ses premiers pas une brochette de grands bibliothécaires qui ont contribué à l'établissement de ce système moderne de bibliothèques dont le Québec est doté depuis 30 ans: Jean-Rémi Brault, Raymond Boucher, Gabriel C. Allard, Victor Coulombe, Jean-Marie Léveillé, Maurice Auger, parmi plusieurs autres. 
Jean-Rémi Brault, bibliographe inlassable, historien chevronné, homme de fine plume, avec la fougue qu'on lui connaît, joua un rôle déterminant dans la fondation de l'organisme et veilla sur ses premiers pas sans ménager ni son temps, ni sa peine, ni son argent.

Raymond Boucher, homme de vision et d'action, mit son énergie et son prestige au service de l'organisation naissante, travailla à préciser son orientation première et à rallier l'adhésion des collègues des quatre coins du Québec.

Mais le départ et la survie de la jeune Centrale eurent été compromis sans la clairvoyance politique et l'appui fidèle de Jean-Marie Beauchemin qui veilla d'abord à sa naissance sous l'édige de la Fédération des collèges classiques en 1964, puis cautionna son intégration au ministère de l'Éducation en 1967. Depuis, son intérêt et son amitié ne se sont jamais démentis à l'endroit de SDM.

Au début des années 70 , Yves Martin, alors sous-ministre de l'Éducation, donna le feu vert à l'établissement de bibliothèques dans les nouvelles polyvalentes. Sa décision s'appuyait sur la disponibilité des répertoires bibliographiques et catalographiques de la Centrale des bibliothèques qui garantissaient le succès de l'opération. C'est dans ce sillage que prit place l'énergique action de Georges Legendre, grand promoteur des bibliothèques scolaires au Québec.

À la fin de cette même décennie, Roland Arpin, alors sous-ministre adjoint de l'Éducation, se voyait officieusement confier le mandat de démanteler cet encombrant organisme, présumé trop onéreux eu égard aux besoins déclinants et aux moyens qui se raréfiaient dans une mauvaise conjoncture économique. Fidèle à sa méthode, ce dernier se livra à un examen minutieux de la situation et décréta que la Centrale devait plutôt se développer grâce à une informatisation de son fonctionnement et à un élargissement de son rayonnement auprès d'une plus large clientèle.

Le premier directeur général du Service général des moyens d'enseignement, Réal Michaud, résista aux pressions qui s'exerçaient dans le sens d'un démembrement de cette "coûteuse" Centrale. Le deuxième et dernier directeur de ce même service, Jacques Laberge, dressa le constat que la Centrale des bibliothèques était une entreprise qui avait acquis définitivement sa raison d'être et jeta les bases de son statut actuel de corporation sans but lucratif.

On ne saurait passer sous silence le fait que pendant quinze ans, de 1967 à 1982, le Centre d'animation, de développement et de recherche en éducation (CADRE) assuma la gestion administrative et financière de la Centrale, propriété de l'État, en vertu d'un arrangement inédit. Mathieu Girard, à titre de directeur général du CADRE, démontra une gestion à la fois intelligente, prudente, ouverte, et responsable, permettant à la Centrale de remplir sa mission dans le cadre plus souple de l'entreprise privée. Roland Cousineau, fidèle compagnon de la première heure, le seconda en supervisant l'activité financière courante.

Cette brève revue, injuste dans ses silences ou ses oublis, illustre assez bien comment la Centrale des bibliothèques a toujours rencontré sur sa route ces gestionnaires éclairés et cultivés, ces collaborateurs engagés qui l'ont puissamment soutenue aux moments cruciaux de son développement.

\section{Les ailes de la liberté}

À la recherche de sécurité, la Centrale des bibliothèques trouvait un havre protecteur en 1967 grâce à la décision d'incorporation à l'administration publique. Toutefois, l'évidence s'imposa rapidement que ce cadre de fonctionnement ne convenait guère à la nature et aux fins de l'entreprise. Sous peine de s'étioler, elle réclamait une plus grande latitude dans la décision et dans l'action: rapidité de réaction, vivacité du réalignement, marge de manoeuvre, souplesse de fonctionnement, flexibilité administrative et financière.

On trouva une parade dans la signature d'un contrat de gestion avec le CADRE dès 1968; en 1982 , I'incorporation à titre d'organisme sans but lucratif consacra cette liberté retrouvée. Ainsi, assimilée au secteur privé, la Centrale des bibliothèques s'est développée normalement en symbiose étroite avec le milieu, a diversifié ses services et ses marchés, a fait preuve de créativité et de dynamisme dans une évolution soutenue et un rajustement systématique de son activité.

Issue du milieu, résultat d'une forte volonté de concentration, de coopération et de coordination manifestée par la base, la Centrale a traditionnellement entretenu des liens serrés avec sa clientèle. En font foi les nombreux et durables comités de liaison, la tounée annuelle des bibliothèques. Elle demeurera proche de ses racines; le milieu garda une influence prépondérante sur ses orientations.

Un examen méthodique de sa publicité annuelle mettrait en lumière les fréquentes mutations qui ont modifié la configuration de ses services au cours du dernier quart de siècle. Pour un organisme parfois taxé de conservatisme, il est étonnant de constater la capacité de renouvel- 
lement dont il a fait preuve et qui a périodiquement transformé son visage. Le virage technologique constitue évidemment la plus spectaculaire de ses métamorphoses.

Fille légitime des collèges classiques, la Centrale des bibliothèques s'émancipa précocement pour se lier aux bibliothèques scolaires, puis aux nouveaux cégeps, pour courtiser plus tard les bibliothèques universitaires et spécialisées. Finalement, elle aborda le grand public et les entreprises, traita avec les services publics et parapublics. Elle déborda même les frontières du Québec et du Canada et traversa quelques fois les mers.

Son premier mandat recouvrait l'indexation coopérative des revues, mais il ne tarda pas à s'enrichir du choix et du traitement des livres, des documents audiovisuels, des périodiques, des jeux et jouets éducatifs, des logiciels. Sa vocation s'est vite multimédiatisée et elle en vint à occuper significativement un vaste secteur de l'information documentaire de langue française.

\section{Conclusion}

Cette rapide incursion dans le passé, biaisée il est vrai par une vision et une interprétation toute personnelles, aura quelque intérêt si elle réussit à donner un éclairage significatif à la réalité présente de SDM et à inspirer ses choix d'avenir. En soulignant le caractère évolutif de l'entreprise, nous avons sous-entendu que son visage se modifiera encore et que la célébration du $50^{\circ}$ anniversaire nous réserve des surprises.
On peut regretter que la cible de 1963 se soit quelque peu estompée. Le sous-développement chronique des bibliothèques scolaires n'avait guère stimulé l'esprit d'initiative de SDM à leur endroit. Encore que depuis trois ans, l'entreprise suit de près et activement les signes du renouveau qui s'amorce et qui devrait donner sa pleine mesure avec l'application d'un plan triennal du ministère de l'Éducation. SDM y trouvera l'occasion d'une exploitation intensive de ses services et pourra tourner à plein régime.

Mais une entreprise de la taille de SDM devra toujours compter sur la diversification de ses services et de ses marchés pour assurer le pain de l'avenir. Souhaitons qu'elle puisse le faire dans la fidélité à sa vocation première.

Les constantes que nous avons rappelées expliquent en partie la verdeur de SDM. Un développement plus cohérent et mieux maîtrisé, une gestion moderne, à la fois rigoureuse et dynamique, une volonté efficace de liaison avec les milieux d'enseignement, apparaissent maintenant comme l'orientation retenue par la nouvelle direction.

Avec nos meilleurs voeux et nos sincères félicitations à tous les membres de l'équipe de SDM, invoquons comme motif supplémentaire de confiance en l'avenir cette bonne étoile qui a présidé à la naissance de l'entreprise et qui ne lui a jamais fait défaut dans la suite de son histoire.

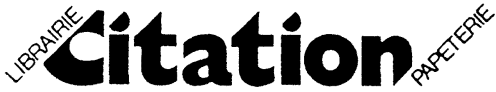

Fournitures de bureau - Papeterie

Mobilier de bureau - Livres

Yvon Daigneault

Dir. Service aux Collectivités

Siège Social

7400 , Boul. Taschereau

(Place Portobello), Brossard

(Québec) J4W $1 \mathrm{M} 9$
Librairie Agréée

Librairie Agréé

(514) 465-0460 